\title{
Erratum to: Effect of Hyaluronidase on the Pharmacokinetics of Free and Total Platinum Species after Intra-Arterial Cisplatin in Refractory Patients with Colorectal Liver Metastases
}

\author{
Dario Civalleri - Mauro Esposito - Franco De Cian • \\ Nadia Balletto • Guido Mondini • Luca Gogioso • \\ Maurizio Viale $\cdot$ Maria O. Vannozzi
}

Published online: 11 March 2014

(c) Springer International Publishing Switzerland 2014

Erratum to: Clin Drug Invest (1996) 12(2): 94-104

DOI 10.2165/00044011-199612020-00005

Title page: Author affiliation which previously read

Dario Civalleri, Mauro Esposito, Franco DeCian, Nadia Balletto, Guido Mondini, Luca Gogioso, Maurizio Viale and Maria O. Vannozzi

\section{Should read}

Dario Civalleri, Mauro Esposito, Franco De Cian, Nadia Balletto, Guido Mondini, Luca Gogioso, Maurizio Viale and Maria O. Vannozzi

The third author's given name is Franco and the family name is De Cian.

The online version of the original article can be found under doi:10.2165/00044011-199612020-00005.

D. Civalleri · F. De Cian · N. Balletto · G. Mondini Istituto di Clinica Chirurgica I, Università di Genova, Genova, Italy

M. Esposito $(\bowtie) \cdot$ M. Viale $\cdot$ M. O. Vannozzi Servizio di Farmacologia Tossicologica, Istituto Nazionale per la Ricerca sul Cancro, 1ST, Largo Rosanna Benzi 10, I-16132 Genova, Italy

L. Gogioso Centro di Informatica, Istituto Nazionale per la Ricerca sul

Cancro, Genova, Italy 